\title{
Perfusion-Based Selection for Endovascular Reperfusion Therapy in Anterior Circulation Acute Ischemic Stroke
}

\author{
S. Prabhakaran, M. Soltanolkotabi, A.R. Honarmand, R.A. Bernstein, V.H. Lee, J.J. Conners, F. Dehkordi-Vakil,
}

A. Shaibani, M.C. Hurley, and S.A. Ansari

\begin{abstract}
BACKGROUND AND PURPOSE: Controversy exists about the role of perfusion imaging in patient selection for endovascular reperfusion therapy in acute ischemic stroke. We hypothesized that perfusion imaging versus noncontrast CT- based selection would be associated with improved functional outcomes at 3 months.
\end{abstract}

MATERIALS AND METHODS: We reviewed consecutive patients with anterior circulation strokes treated with endovascular reperfusion therapy within 8 hours and with baseline NIHSS score of $\geq 8$. Baseline clinical data, selection mode (perfusion versus NCCT), angiographic data, complications, and modified Rankin Scale score at 3 months were collected. Using multivariable logistic regression, we assessed whether the mode of selection for endovascular reperfusion therapy (perfusion-based versus NCCT-based) was independently associated with good outcome.

RESULTS: Two-hundred fourteen patients (mean age, 67.2 years; median NIHSS score, 18; MCA occlusion 74\% and ICA occlusion 26\%) were included. Perfusion imaging was used in 76 (35.5\%) patients (39 CT and 37 MR imaging). Perfusion imaging-selected patients were more likely to have good outcomes compared with NCCT-selected patients ( 55.3 versus $33.3 \%, P=.002$ ); perfusion selection by CT was associated with similar outcomes as that by MR imaging (CTP, 56.; MR perfusion, 54.1\%; $P=.836$ ). In multivariable analysis, CT or MR perfusion imaging selection remained strongly associated with good outcome (adjusted OR, 2.34; $95 \% \mathrm{Cl}, 1.22-4.47$ ), independent of baseline severity and reperfusion.

CONCLUSIONS: In this multicenter study, patients with acute ischemic stroke who underwent perfusion imaging were more than 2-fold more likely to have good outcomes following endovascular reperfusion therapy. Randomized studies should compare perfusion imaging with NCCT imaging for patient selection for endovascular reperfusion therapy.

ABBREVIATIONS: DEFUSE-2 = Diffusion and Perfusion Imaging Evaluation for Understanding Stroke Evolution Study 2; ERT = endovascular reperfusion therapy; MRP = MR perfusion; THRIVE $=$ Totaled Health Risks in Vascular Events

E ndovascular reperfusion therapy (ERT) for acute ischemic stroke has been associated with mixed results. In trials of carefully selected patients with middle cerebral artery occlusion, a benefit of intra-arterial thrombolysis over placebo was seen when patients were treated within 6 hours. ${ }^{1,2}$ However, subsequent single-arm studies of mechanical embolectomy have observed less impressive results ${ }^{3,4}$ and suggest that outcomes are related to several key factors, including patient characteristics (age, co-morbid-

Received October 9, 2013; accepted after revision November 25.

From the Departments of Neurology (S.P., R.A.B.) and Radiology (M.S., A.R.H., A.S., M.C.H., S.A.A.), Northwestern University, Chicago, Illinois; and Department of Neurology (V.H.L., J.J.C.), Rush University Medical Center, Chicago, Illinois; and Department of Economics and Decision Sciences (F.D.-V.), Western Illinois University, Macomb, Illinois.

Please address correspondence to Shyam Prabhakaran, MD, MS, Northwestern University-Feinberg School of Medicine, 710 N Lake Shore Dr, Room 1417, Chicago, IL 60611; e-mail: shyam.prabhakaran@northwestern.edu

三 Indicates article with supplemental on-line appendix

http://dx.doi.org/10.3174/ajnr.A3889 ities, and stroke severity) and treatment factors (time to reperfusion). ${ }^{5-10}$ Radiographic features, including pretreatment tissue status by NCCT of the head, brain MR imaging, and perfusion imaging (CTP or MR perfusion [MRP]), may improve patient selection. ${ }^{5,8,9,11}$ Few studies have compared NCCT-based selection with perfusion imaging-based selection of patients for ERT following acute ischemic stroke. ${ }^{12,13} \mathrm{We}$, therefore, sought to compare NCCT selection with perfusion imaging selection as a predictor of good outcome following ERT. We hypothesized that perfusion imaging-based selection would be associated with better functional outcomes at 3 months compared with NCCT-based selection alone.

\section{MATERIALS AND METHODS \\ Data Sources}

We analyzed a retrospective registry of consecutive patients treated with endovascular therapy at 4 tertiary stroke centers from January 2007 to December 2012. Participating hospitals submit- 
ted information on consecutive patients with ischemic stroke treated with ERT, de-identified for compliance with the Health Insurance Portability and Accountability Act. Institutional review board approval was granted from each participating center.

Inclusion criteria for this study were patients with acute ischemic stroke who presented within 8 hours of symptom onset with anterior circulation large-vessel occlusions and pretreatment $\mathrm{Na}$ tional Institutes of Health Stroke Scale score of $\geq 8$. Data were analyzed regarding demographics, previous medical history, pretreatment stroke severity by NIHSS score, time of symptom onset, treatment with intravenous tissue plasminogen activator, mode of selection for ERT (NCCT versus CTP or MRP), time of groin puncture, location of arterial occlusion on angiography, reperfusion status, radiographic interpretation of hemorrhages, and clinical outcomes. The site of occlusion was determined angiographically as the most proximal ipsilateral lesion with a TICI 0 or 1 perfusion grade. Successful reperfusion was defined as TICI $2 b$ or higher on the final angiographic image. ${ }^{14}$ Symptomatic hemorrhage was defined as a parenchymal hematoma type 1 or 2 by using the European Cooperative Acute Stroke Study definition ${ }^{15}$ associated with an increase of $>4$ in the NIHSS score and any wire perforation resulting in subarachnoid hemorrhage. Patients with an mRS score of $\leq 2$ at 90 days were considered to have good clinical outcomes. Pretreatment Totaled Health Risks in Vascular Events (THRIVE) scores were calculated on the basis of published criteria. ${ }^{6}$ The THRIVE score has been validated as a simple pretreatment scoring tool to predict clinical outcome, mortality, and symptomatic hemorrhage after ERT and is calculated on the basis of age, NIHSS score, and history of atrial fibrillation, diabetes, and hypertension.

\section{Imaging Acquisition and Interpretation}

Site-specific imaging acquisition and software application details are included in the On-line Appendix. Imaging-based patient selection for ERT was dependent on local site determination of CT, CTP, or MRP eligibility criteria. Both qualitative visual inspection and volumetric measurements were used at sites. General imaging inclusion criteria were based on published or consensus guidelines: 1) NCCT with less than one-third hypoattenuation in the MCA territory; or 2) core infarct volume with less than one-third of the MCA territory or $<70 \mathrm{~mL}$, and perfusion abnormality to core infarct mismatch ratio of $>1.2$, which was increased to 1.8 after 2009, consistent with the Diffusion and Perfusion Imaging Evaluation for Understanding Stroke Evolution Study 2 (DEFUSE-2) trial. $^{16}$ Sites used similar relative and absolute thresholds for the definition of core infarct (cerebral blood volume of $<2 \mathrm{~mL} / 100 \mathrm{~g}$ on CTP or DWI hyperintensity with decreased ADC on MR imaging) and abnormal perfusion to estimate the ischemic penumbra (MTT $>6$ seconds on CTP or timeto-maximum of the tissue residue function $>6$ seconds on MRP). However, these guidelines were used by neurointerventional physicians at each site without central adjudication.

In a post hoc analysis of perfusion-based selected cases, we calculated mismatch ratios of abnormal perfusion volume to core infarct volume. Determinations of the core infarct and penumbra volumes were performed by using the $\mathrm{ABC} / 2$ method on postprocessing software (On-line Appendix) at each site. ${ }^{17}$ Two indepen- dent raters also performed measurements in a sample of $10 \mathrm{de}$ identified CTP and MRP scans, respectively, and demonstrated high reproducibility (CTP: Kendall $\tau=0.822, P=.002$; MRP: Kendall $\tau=0.810, P=.016)$.

\section{Endovascular Reperfusion Therapy}

Following transfemoral arterial access and modest (2000-4000 $\mathrm{U}$ ) anticoagulation with heparin, Merci (14X or $18 \mathrm{~L}$; Concentric Medical, Mountain View, California) or Penumbra reperfusion (041/054; Penumbra, Alameda, California) catheters were advanced coaxially over 021/032-inch microcatheters and/or 0.014/ .016-inch microwires to the thromboembolic occlusion per published methods for clot retrieval or aspiration ${ }^{3,4}$; stent retrievers were used in a minority of cases because these were available only after 2011. ${ }^{18,19}$ Intra-arterial thrombolysis with tPA (Genentech, San Francisco, California) was performed as an adjunct with mechanical thrombectomy by embedding the microcatheter in the clot and by using a pulse-spray technique. Major indications for intra-arterial tPA use were partial ineffectiveness of retrieval/aspiration, intraprocedural clot fragmentation with distal migration, or concomitant distal thromboemboli.

\section{Statistical Analysis}

Using univariable tests, we compared demographic, clinical, and outcome data among NCCT- and perfusion-selected patients by using $\chi^{2}$ (or the Fisher exact if appropriate) tests for categoric variables and $t$ tests (or Mann-Whitney if appropriate) for continuous variables. We then assessed univariable associations between these factors and good clinical outcome after ERT (defined as an mRS score of $0-2$ at 90 days). A multivariable logistic regression analysis was performed to identify independent predictors of good outcome. Candidate variables were selected on the basis of statistically significant univariable relationships with good outcome. The fitness of the model was tested by using the Hosmer-Lemeshow test. All $P$ values are 2 -sided, with $P<.05$ considered statistically significant. Analyses were performed by using the Statistical Package for the Social Sciences software (Version 21; IBM, Armonk, New York).

\section{RESULTS}

Among 262 patients treated with ERT at 4 tertiary hospitals in the Chicago region, 48 were excluded due to vertebrobasilar artery occlusion $(n=32)$, isolated anterior cerebral artery occlusion $(n=3)$, isolated posterior cerebral artery occlusion $(n=1)$, initial NIHSS $<8(n=5)$, treatment $>8$ hours from symptom onset $(n=4)$, and missing outcome data $(n=3)$. Among the 214 patients who met our inclusion criteria (mean age, $67.2 \pm 16.0$ years; male, $40.7 \%$ ), the median NIHSS score was 18 with $74.3 \%$ presenting with MCA occlusions, while $25.7 \%$ had occlusions involving the ICA and MCA.

Seventy-six (35.5\%) patients were selected by using perfusion imaging (CTP: 39, 18.2\%; MRP: 37, 17.3\%). Selection mode varied by hospital with sites contributing $10.5 \%-34.2 \%$ of perfusionselected cases $(P<.001)$. During the study period, the use of perfusion imaging selection increased from $21.1 \%$ in year 1 to $56.5 \%$ in year $6(P=.215)$. Median onset to groin puncture was 311 (interquartile range, 242-380) minutes but improved during 
Table 1: Univariable analysis of characteristics associated with perfusion imaging selection

\begin{tabular}{|c|c|c|c|}
\hline & $\begin{array}{l}\text { Perfusion Selection } \\
\qquad(n=76)\end{array}$ & $\begin{array}{l}\text { NCCT Selection } \\
\quad(n=138)\end{array}$ & $P$ Value \\
\hline Age (yr) & & & $<.001$ \\
\hline Mean (SD) & $72.4(14.1)$ & $64.4(16.3)$ & \\
\hline Male, No. $(\%)$ & $26(34.2)$ & $61(44.2)$ & .154 \\
\hline Onset to arrival time (min) & & & .520 \\
\hline Median (IQR) & $245(123.5-300.0)$ & $241.5(180.0-320.0)$ & \\
\hline Initial NIHSS score & & & .018 \\
\hline Median (IQR) & $18(13-20)$ & $19(16-22)$ & \\
\hline Initial THRIVE score & & & .529 \\
\hline Median (IQR) & $4(3-5)$ & $4.5(3-6)$ & \\
\hline IV tPA prior to ERT, No. (\%) & $31(40.8)$ & $67(48.6)$ & .275 \\
\hline Location of occlusion, No. (\%) & & & $<.001$ \\
\hline Middle cerebral artery & $68(89.5)$ & $91(65.9)$ & \\
\hline Internal carotid artery & $8(10.5)$ & $47(34.1)$ & \\
\hline Mode of IA therapy & & & .028 \\
\hline Lytic only & $3(3.8)$ & 0 & \\
\hline Mechanical only & $44(57.9)$ & $95(68.8)$ & \\
\hline Lytic + mechanical & $29(38.2)$ & $43(31.2)$ & \\
\hline Onset-to-GP time in minutes & & & .366 \\
\hline Median (IQR) & $348(225.75-399.75)$ & 309 (245.5-375.0) & \\
\hline Symptomatic hemorrhage, No. (\%) & $4(5.3)$ & $17(12.3)$ & .148 \\
\hline $\mathrm{TICl}$ 2b/3 reperfusion, No. (\%) & $46(62.5)$ & $65(47.1)$ & .036 \\
\hline Year & & & .215 \\
\hline 2007 & $4(5.3)$ & 15 (10.9) & \\
\hline 2008 & 9 (11.8) & 22 (15.9) & \\
\hline 2009 & 18 (23.7) & $29(21.0)$ & \\
\hline 2010 & $17(22.4)$ & $35(25.4)$ & \\
\hline 2011 & 15 (19.7) & $27(19.6)$ & \\
\hline 2012 & 13 (17.1) & $10(7.2)$ & \\
\hline
\end{tabular}

Note:-IQR indicates interquartile range; IA, intra-arterial; GP, groin puncture.

the study period (year 1: 350 minutes to year 6:228 minutes; $P=$ .060). Mechanical thrombectomy devices were commonly used and often in combination: Merci $(n=106)$, Penumbra $(n=$ $160)$, and stent retrievers $(n=15)$. TICI 2 b or 3 reperfusion was achieved in $52.4 \%$ and did not vary by site (range, $45.9 \%-$ $69.8 \% ; P=.081)$ or with time $(P=.280)$. Symptomatic hemorrhage occurred in $9.8 \%$ of patients and did not vary by site (range, 3.0\%-13.3\%; $P=.349$ ). Good outcome was observed in $41.1 \%$ of patients at 3 months. The mortality rate at 3 months was $16.4 \%$.

When we compared those patients selected by perfusion imaging with those selected by NCCT alone, perfusion imagingselected patients were older, had slightly lower NIHSS scores, more frequently had MCA occlusions and received multimodal (lytic plus mechanical) intra-arterial therapies, and had higher rates of TICI $2 \mathrm{~b}$ or 3 reperfusion after treatment (Table 1). Other factors such as receipt of IV tPA before ERT, onset-to-arrival and onset-to-groin puncture times, THRIVE score, and symptomatic hemorrhage rates were similar between groups.

Perfusion-selected patients were more likely to have good outcomes at 3 months compared with NCCT-selected patients (any perfusion: $55.3 \%$ [CTP: $56.4 \%$ and MRP 54.1\%] versus NCCT: $33.3 \%, P=.002)$. Good outcome among perfusion-selected patients ranged from $42.3 \%$ to $70.8 \%$ across the 4 sites $(P=.213)$. Perfusion-selection patients also had lower 3-month mortality rates $(7.9 \%$ versus $21.0 \%, P=.012)$. In post hoc analyses of patients selected by perfusion imaging, the median core infarct volume was $15.7 \mathrm{~mL}$ (interquartile range, $5.6-41.7 \mathrm{~mL}$ ), and ischemic penumbra volume was $103.8 \mathrm{~mL}$ (interquartile range, 64.7-
$154.0 \mathrm{~mL}$ ). The median mismatch ratio was 5.8 (interquartile range, 2.5-17.6). Only $9(11.8 \%)$ of the perfusion-selected patients had mismatch ratios of $<1.8$.

Those achieving good outcome also had lower NIHSS and THRIVE scores, were more likely to have received IV tPA before ERT, had TICI $2 \mathrm{~b}$ or 3 reperfusion after treatment, and were less likely to have symptomatic hemorrhages (Table 2). Other factors, including the location of arterial occlusion and mode of therapy, were similarly distributed by outcome.

In multivariable analysis (Table 3 ) to identify predictors of good outcome (mRS 0-2) and adjusting for factors significantly associated with the mode of selection or with outcome (age, calendar year, initial NIHSS score, IV tPA use, location of occlusion, mode of therapy, final TICI, symptomatic hemorrhage), perfusion selection remained independently associated with good outcome (adjusted OR, 2.3; 95\%, CI $1.23 \%-4.47 \%)$.

\section{DISCUSSION}

In a multicenter study, we observed that patients selected for ERT by perfusion imaging were more than 2-fold more likely to have good functional outcomes compared with NCCT-selected patients, despite an older age and modest delays in time to treatment. These results were independent of other known predictors of good outcome, including stroke severity, comorbidities, IV tPA utilization, and TICI reperfusion score.

Our data are consistent with some previously published work that observed higher rates of good outcome ranging from $41 \%$ to $67 \%$ by using perfusion selection for ERT compared with historic controls. ${ }^{20}$ Others have noted that perfusion selection attenuates or negates the influence of time so that good outcomes can be achieved in similar proportions after 8 hours compared with $<8$ hours by using perfusion imaging. ${ }^{21,22}$ However, 2 retrospective prior studies comparing perfusion selection with NCCT selection have failed to show a benefit of one approach over the other. ${ }^{12,13}$ Hassan et $\mathrm{al}^{13}$ demonstrated equivalent discharge outcomes, symptomatic hemorrhage rates, and mortality rates in a retrospective analysis comparing NCCT- versus CTP-based patient selection. The methodology for qualitative and quantitative penumbral assessment was MTT $\geq 20 \%$ of the affected region or a presumed mismatch ratio of $>1.2$. Sheth et $\mathrm{al}^{12}$ also performed a multicenter retrospective analysis and found no difference in clinical outcomes with NCCT, CTP, or MR imaging-based selection. However, this study provided no specific parameters used for advanced imaging selection and likely lacked standardized patientselection approaches in each subgroup. Neither study performed post hoc core infarct/penumbra analyses. Lack of standardized and/or less stringent perfusion imaging criteria may explain their 
Table 2: Univariable analysis of characteristics associated with good outcome (mRS 0-2) at 3 months

\begin{tabular}{|c|c|c|c|}
\hline & $\begin{array}{l}\text { Good Outcome } \\
\qquad(n=88)\end{array}$ & $\begin{array}{l}\text { Poor Outcome } \\
\quad(n=126)\end{array}$ & $P$ Value \\
\hline Age (yr) & & & .891 \\
\hline Mean (SD) & $67.0(15.6)$ & $67.4(16.3)$ & \\
\hline Male, No. (\%) & $36(40.5)$ & $51(40.9)$ & .949 \\
\hline Onset-to-arrival time in minutes & & & .627 \\
\hline Median (IQR) & $240(139.0-304.5)$ & $250(178.5-366.0)$ & \\
\hline Initial NIHSS score & & & .009 \\
\hline Median (IQR) & $18(12.25-21.0)$ & $19(16-22)$ & \\
\hline Initial THRIVE score & & & .004 \\
\hline Median (IQR) & $4(3-5)$ & $5(4-6)$ & \\
\hline IV tPA prior to ERT, No. (\%) & 49 (55.7) & 49 (38.9) & .015 \\
\hline Location of occlusion, No. (\%) & & & .607 \\
\hline Middle cerebral artery & $67(76.1)$ & $92(73.0)$ & \\
\hline Internal carotid artery & $21(23.9)$ & $34(27.0)$ & \\
\hline Mode of ERT & & & .488 \\
\hline Lytic only & $2(2.3)$ & $1(0.8)$ & \\
\hline Mechanical only & $54(61.4)$ & $85(67.5)$ & \\
\hline Lytic + mechanical & $32(44.4)$ & $40(31.7)$ & \\
\hline Onset to GP time in minutes & & & .790 \\
\hline Median (IQR) & $306.5(240.0-385.0)$ & 315 (242.75-379.75) & \\
\hline Symptomatic hemorrhage, No. (\%) & $3(3.4)$ & $18(14.3)$ & .009 \\
\hline $\mathrm{TICl} 2 \mathrm{~b} / 3$ reperfusion, No. (\%) & 64 (72.7) & $47(37.9)$ & $<.001$ \\
\hline Year & & & .147 \\
\hline 2007 & $7(8.0)$ & $12(9.5)$ & \\
\hline 2008 & 10 (11.4) & $21(9.8)$ & \\
\hline 2009 & $21(23.9)$ & $26(20.6)$ & \\
\hline 2010 & $29(33.0)$ & 23 (18.3) & \\
\hline 2011 & $13(14.0)$ & $29(23.0)$ & \\
\hline 2012 & $8(9.1)$ & 15 (11.9) & \\
\hline
\end{tabular}

Note:- $\mathrm{IQR}$ indicates interquartile range; GP, groin puncture.

Table 3: Multivariable model for good outcome (mRS 0-2 at 3 months) with the following dependent variables: age, calendar year, initial NIHSS score, IV tPA use, selection mode, location of occlusion, mode of therapy, final TICl score, and symptomatic hemorrhage ${ }^{a}$

\begin{tabular}{lccc}
\hline & Adjusted OR & $\mathbf{9 5 \%} \mathrm{Cl}$ & $\boldsymbol{P}$ Value \\
\hline Selection mode & & & .010 \\
NCCT only (reference) & - & - & \\
Perfusion imaging & 2.34 & $1.23-4.47$ & \\
Initial NIHSS score (per point) & 0.92 & $0.87-0.98$ & .006 \\
IV tPA prior to IAT & 2.12 & $1.13-3.96$ & .019 \\
TICl score & & & $<.001$ \\
TICl $<2$ b (reference) & - & - & \\
$\mathrm{TICl} 2 \mathrm{~b}$ or 3 & 4.26 & $2.27-8.03$ & \\
\hline
\end{tabular}

Note:-IQR indicates interquartile range; IAT, intra-arterial therapy.

${ }^{a}$ Hosmer-Lemeshow test for goodness of fit: $P=.229$.

null results compared with our findings. Additionally, Sheth et $\mathrm{al}^{12}$ noted that CT perfusion or MR imaging selection added approximately a 60-minute delay to treatment. Although we also observed a 40-minute delay with perfusion selection, the potential improved selection and association with better outcomes may justify this approach. It should be acknowledged that perfusionbased selection may result in higher proportions of treated patients with good outcomes, though fewer patients overall may actually receive ERT compared with NCCT-based selection.

Studies of imaging-based selection in acute ischemic stroke have produced mixed results, at least partly due to the variability of techniques, postprocessing software algorithms, and definitions of core infarct and penumbra that parse oligemia from true ischemia. ${ }^{23}$ Critics of perfusion-based patient-selection strategies for acute stroke intervention cite the limited MR imaging acces- sibility; additional time required for imaging, resulting in endovascular treatment delays; the lack of standardized postprocessing perfusion software; failure to quantitatively model the dynamic properties of in vivo cerebral perfusion (ie, contrast delay-dispersion correction and retrograde pial collateral supply); and an inability to differentiate a true penumbra (ischemic tissue destined to infarct without reperfusion) versus false penumbra (oligemic tissue that will survive). Furthermore, a measurement made 1 or 2 hours before reperfusion may not be a reliable indicator of tissue fate; real-time measurement of tissue perfusion at the time of reperfusion therapy is preferred.

Prior clinical trials of perfusionbased selection for intravenous and intra-arterial reperfusion therapies have produced mixed results. In the desmoteplase studies, the phase 3 trial showed no benefit of delayed thrombolysis based on perfusion imaging selection, defined as a mismatch (penumbra-to-infarct) ratio of $>1.2$, despite promising phase 2 study results. ${ }^{24-26}$ However, post hoc analyses suggested that a minimal mismatch volume of $60 \mathrm{~mL}$ would have led to a benefit in favor of desmoteplase. ${ }^{27}$ Other intravenous thrombolytic phase 2 trials confirmed potential benefit in delayed treatment by using MR diffusion-perfusion imaging-based patient selection. ${ }^{28,29}$ In the DEFUSE-2 trial, endovascular reperfusion was associated with a 5 -fold increase in favorable clinical outcomes among patients with target mismatch (defined as ratio of $>1.8$ ), while no benefit was seen among patients without target mismatch. The trial used standardized MR imaging postprocessing software (RAPID; iSchemaView Inc, Palo Alto, California), which allowed uniform definitions of core infarct, severe perfusion abnormalities, and hence possibly a more accurate measurement of penumbra. ${ }^{16}$ Our data are consistent with the DEFUSE-2 study, in which large mismatch ratios (median, 5.8 in our study) were also observed. These data suggest that perfusion imaging selection, if optimized with standardized parameters that best define mismatch and true penumbra, may identify patients most likely to benefit while excluding those who may incur harm from reperfusion therapy.

In contrast, the recently reported Mechanical Retrieval and Recanalization of Stroke Clots Using Embolectomy trial found no benefit of ERT over medical treatment, irrespective of a favorable perfusion imaging profile ("penumbral" versus "nonpenumbral" pattern). ${ }^{30}$ Most interesting, patients with a penumbral pattern were found to have better outcomes and smaller infarct growth, suggesting a protective effect. It is therefore possible that penumbral selection, as in our study, identifies those patients destined for better outcomes irrespective of treatment. The Mechanical Retrieval and Recanalization of Stroke Clots Using Embolectomy 
trial identified significant delays in randomization, ranging from 5 to 6 hours, suggesting relatively late CTP/MRP acquisitions. Penumbral patterns noted at various time points after stroke onset (early versus late) may also be indicative of differing risk profiles so that early imaging may mark those with a high risk of infarct growth and deterioration, while late imaging may identify those with good collateral flow that protects against infarct growth and deterioration. ${ }^{31,32}$ Further work related to imaging techniques with respect to reproducibility, standardized definitions of optimal parameters, and postprocessing methodologies for distinguishing penumbra from infarct core are clearly needed.

Our study has several limitations. First, we did not prospectively adjudicate NCCT or perfusion imaging selection for ERT. While ASPECTS and mismatch criteria have been developed and may have been used at our sites, ${ }^{11,16}$ decisions regarding eligibility were determined by the local treating physicians by using differing modalities and software platforms. In addition, CTP and MRP may have differing accuracies for predicting infarct core and penumbra and differing spatial coverage so that CTP may provide only limited coverage ${ }^{23}$; however, outcomes among those selected by CT versus MR perfusion were not different in our study. Second, our results are not generalizable to posterior circulation strokes. Besides different natural histories compared with anterior circulation stroke, posterior circulation stroke has challenges in perfusion imaging selection. Only small studies using MRP have been conducted in posterior circulation stroke, and none has clearly demonstrated its utility in selection for ERT. ${ }^{33}$ Third, outcomes following ERT are also dependent on time to reperfusion. ${ }^{10}$ Capturing reperfusion times is often challenging in clinical practice due to infrequent sequential angiographic runs and the possibility of slow and steady reperfusion or partial followed by complete reperfusion. Fourth, the type of mechanical thrombectomy device was not controlled for in the study, though a minority (15 patients) was treated with the new generation of stent retrievers. Recent advances in retrievable-stent technology may lead to earlier and higher rates of reperfusion with minimal symptomatic hemorrhage. ${ }^{18,19}$

Fifth, all data in the registry were entered by local sites without central adjudication and were aggregated post hoc in a de-identified manner. We do not know how many patients were excluded from ERT on the basis of NCCT or perfusion imaging results; therefore, we cannot comment on outcomes in those who did not receive ERT. Grading TICI scores, in particular, can demonstrate significant interobserver variability, depending on whether the primary arterial occlusion was completely or partially recanalized, the presence of distal emboli, and the role of pial collaterals. Grading final reperfusion can also demonstrate site-reported bias in favor of better scores, compared with central adjudication, as demonstrated in the solitaire flow restoration device versus the Merci retriever in patients with acute ischaemic stroke (SWIFT) trial. ${ }^{19}$ However, our hospitals have participated in large endovascular clinical trials, and consensus definitions were used to define key variables such as TICI grade and symptomatic hemorrhage. Angiographic collateral grade was also not systematically documented or available on post hoc review. Sixth, bias from the treating physicians may have resulted in use of perfusion selection for specific subgroups of patients, which could account for the differ- ence in outcomes. Seventh, we also did not adjust for site characteristics such as volume of cases per year or operator experience, which may influence outcomes. ${ }^{34}$ However, site performance as measured by rates of TICI $2 \mathrm{~b}$ or 3 reperfusion and symptomatic hemorrhage were not different by site. Last, residual or unmeasured confounding could account for some or all of our results.

\section{CONCLUSIONS}

In a multicenter study, we observed nearly double the rates of good outcome following ERT for acute ischemic stroke among patients selected by perfusion imaging compared with NCCT alone. Nevertheless, the added "costs" in terms of time delays, imaging acquisition and interpretation, and health care resource use need to be carefully considered. Quality improvement efforts in light of the recent endovascular stroke trials should focus on strategies to reduce throughput delays in onset-to-treatment times, especially with perfusion-based selection.

Disclosures: Shyam Prabhakaran—UNRELATED: Consultancy: American Heart Association as medical director of Quality Enhancement for Speedy Thrombolysis in Stroke initiative, Grants/Grants Pending: National Institute of Neurological Disorders and Stroke, Royalties: Up-To-Date Inc.

\section{REFERENCES}

1. Furlan A, Higashida R, Wechsler L, et al. Intra-arterial prourokinase for acute ischemic stroke. The PROACT II study: a randomized controlled trial. Prolyse in Acute Cerebral Thromboembolism. JAMA 1999;282:2003-11

2. Ogawa A, Mori E, Minematsu K, et al. Randomized trial of intraarterial infusion of urokinase within 6 hours of middle cerebral artery stroke: the Middle Cerebral Artery Embolism Local Fibrinolytic Intervention Trial (MELT) Japan. Stroke 2007;38:2633-39

3. Smith WS, Sung G, Starkman S, et al. Safety and efficacy of mechanical embolectomy in acute ischemic stroke: results of the MERCI trial. Stroke 2005;36:1432-38

4. Penumbra Pivotal Stroke Trial Investigators. The Penumbra Pivotal Stroke Trial: safety and effectiveness of a new generation of mechanical devices for clot removal in intracranial large vessel occlusive disease. Stroke 2009;40:2761-68

5. Fargen KM, Chaudry I, Turner RD, et al. A novel clinical and imaging based score for predicting outcome prior to endovascular treatment of acute ischemic stroke. J Neurointerv Surg 2013;5(suppl 1):i38-43

6. Flint AC, Cullen SP, Faigeles BS, et al. Predicting long-term outcome after endovascular stroke treatment: the Totaled Health Risks in Vascular Events score. AJNR Am J Neuroradiol 2010;31:1192-96

7. Hallevi $\mathrm{H}$, Barreto $\mathrm{AD}$, Liebeskind DS, et al. Identifying patients at high risk for poor outcome after intra-arterial therapy for acute ischemic stroke. Stroke 2009;40:1780-85

8. Vora NA, Shook SJ, Schumacher HC, et al. A 5-item scale to predict stroke outcome after cortical middle cerebral artery territory infarction: validation from results of the Diffusion and Perfusion Imaging Evaluation for Understanding Stroke Evolution (DEFUSE) Study. Stroke 2011;42:645-49

9. Yoo AJ, Verduzco LA, Schaefer PW, et al. MRI-based selection for intraarterial stroke therapy: value of pretreatment diffusion-weighted imaging lesion volume in selecting patients with acute stroke who will benefit from early recanalization. Stroke 2009;40:2046-54

10. Khatri P, Abruzzo T, Yeatts SD, et al. Good clinical outcome after ischemic stroke with successful revascularization is time-dependent. Neurology 2009;73:1066-72

11. Hill MD, Rowley HA, Adler F, et al. Selection of acute ischemic stroke patients for intra-arterial thrombolysis with pro-urokinase by using ASPECTS. Stroke 2003;34:1925-31

12. Sheth KN, Terry JB, Nogueira RG, et al. Advanced modality imaging evaluation in acute ischemic stroke may lead to delayed endovascu- 
lar reperfusion therapy without improvement in clinical outcomes. J Neurointerv Surg 2013;5(suppl 1):i62-65

13. Hassan AE, Zacharatos H, Rodriguez GJ, et al. A comparison of computed tomography perfusion-guided and time-guided endovascular treatments for patients with acute ischemic stroke. Stroke 2010;41:1673-78

14. Higashida RT, Furlan AJ, Roberts $\mathrm{H}$, et al. Trial design and reporting standards for intra-arterial cerebral thrombolysis for acute ischemic stroke. Stroke 2003;34:e109-137

15. Hacke W, Kaste M, Bluhmki E, et al. Thrombolysis with alteplase 3 to 4.5 hours after acute ischemic stroke. $N$ Engl J Med 2008;359:1317-29

16. Lansberg MG, Straka M, Kemp S, et al. MRI profile and response to endovascular reperfusion after stroke (DEFUSE 2): a prospective cohort study. Lancet Neurol 2012;11:860-67

17. Sims JR, Gharai LR, Schaefer PW, et al. ABC/2 for rapid clinical estimate of infarct, perfusion, and mismatch volumes. Neurology 2009;72:2104-10

18. Nogueira RG, Lutsep HL, Gupta R, et al. Trevo versus Merci retrievers for thrombectomy revascularisation of large vessel occlusions in acute ischaemic stroke (TREVO 2): a randomised trial. Lancet 2012;380:1231-40

19. Saver JL, Jahan R, Levy EI, et al. Solitaire flow restoration device versus the Merci retriever in patients with acute ischaemic stroke (SWIFT): a randomised, parallel-group, non-inferiority trial. Lancet 2012;380:1241-49

20. Rai AT, Raghuram K, Domico J, et al. Pre-intervention triage incorporating perfusion imaging improves outcomes in patients undergoing endovascular stroke therapy: a comparison with the device trials. J Neurointerv Surg 2013;5:121-27

21. Abou-Chebl A. Endovascular treatment of acute ischemic stroke may be safely performed with no time window limit in appropriately selected patients. Stroke 2010;41:1996-2000

22. Jovin TG, Liebeskind DS, Gupta R, et al. Imaging-based endovascular therapy for acute ischemic stroke due to proximal intracranial anterior circulation occlusion treated beyond $\mathbf{8}$ hours from time last seen well: retrospective multicenter analysis of 237 consecutive patients. Stroke 2011;42:2206-11

23. Goyal M, Menon BK, Derdeyn CP. Perfusion imaging in acute ischemic stroke: let us improve the science before changing clinical practice. Radiology 2013;266:16-21
24. Furlan AJ, Eyding D, Albers GW, et al. Dose Escalation of Desmoteplase for Acute Ischemic Stroke (DEDAS): evidence of safety and efficacy 3 to 9 hours after stroke onset. Stroke 2006;37:1227-31

25. Hacke W, Albers G, Al-Rawi Y, et al. The Desmoteplase in Acute Ischemic Stroke Trial (DIAS): a phase II MRI-based 9-hour window acute stroke thrombolysis trial with intravenous desmoteplase. Stroke 2005;36:66-73

26. Hacke W, Furlan AJ, Al-Rawi Y, et al. Intravenous desmoteplase in patients with acute ischaemic stroke selected by MRI perfusiondiffusion weighted imaging or perfusion CT (DIAS-2): a prospective, randomised, double-blind, placebo-controlled study. Lancet Neurol 2009;8:141-50

27. Warach S, Al-Rawi Y, Furlan AJ, et al. Refinement of the magnetic resonance diffusion-perfusion mismatch concept for thrombolytic patient selection: insights from the desmoteplase in acute stroke trials. Stroke 2012;43:2313-18

28. Albers GW, Thijs VN, Wechsler L, et al. Magnetic resonance imaging profiles predict clinical response to early reperfusion: the diffusion and perfusion imaging evaluation for understanding stroke evolution (DEFUSE) study. Ann Neurol 2006;60:508-17

29. Davis SM, Donnan GA, Parsons MW, et al. Effects of alteplase beyond $3 \mathrm{~h}$ after stroke in the Echoplanar Imaging Thrombolytic Evaluation Trial (EPITHET): a placebo-controlled randomised trial. Lancet Neurol 2008;7:299-309

30. Kidwell CS, Jahan R, Gornbein J, et al. A trial of imaging selection and endovascular treatment for ischemic stroke. $N$ Engl $\mathrm{J} \mathrm{Med}$ 2013;368:914-23

31. Bang OY, Saver JL, Kim SJ, et al. Collateral flow predicts response to endovascular therapy for acute ischemic stroke. Stroke 2011;42:693-99

32. Bang OY, Saver JL, Kim SJ, et al. Collateral flow averts hemorrhagic transformation after endovascular therapy for acute ischemic stroke. Stroke 2011;42:2235-39

33. Ostrem JL, Saver JL, Alger JR, et al. Acute basilar artery occlusion: diffusion-perfusion MRI characterization of tissue salvage in patients receiving intra-arterial stroke therapies. Stroke 2004;35:e30-04

34. Gupta R, Horev A, Nguyen T, et al. Higher volume endovascular stroke centers have faster times to treatment, higher reperfusion rates and higher rates of good clinical outcomes. J Neurointerv Surg 2013;5:294-97 